\title{
TÁBANOS (DIPTERA: TABANIDAE) DE LA SELVA MEDIANA DEL SUR DE YUCATÁN, MÉXICO
}

\author{
Pablo Camilo MANRIQUE-SAIDE, ${ }^{1}$ Ángel Roberto BRICEÑO-UC, ${ }^{1}$ \\ SERgIo IBÁÑEZ-BERNAL ${ }^{2}$ Y CÉSAR ANTONIO SANDOVAL-RUIZ ${ }^{2}$ \\ ${ }^{1}$ Campus de Ciencias Biológicas y Agropecuarias, Universidad Autónoma de Yucatán. km 15.5 \\ carretera Mérida-Xmatkuil. Apdo. Postal 4-116, Itzimná, Mérida, Yucatán, México. \\ ${ }^{2}$ Instituto de Ecología, A. C., Red de Ambiente y Sustentabilidad. Carretera antigua a Coatepec No. \\ 351, Congregación El Haya, Xalapa 91070, Veracruz, México.
}

Manrique-Saide, P. C., Briceño-Uc, A. R., Ibáñez-Bernal, S. \& Sandoval-Ruiz, C. A. 2012. Tábanos (Diptera: Tabanidae) de la selva mediana del sur de Yucatán, México. Acta Zoológica Mexicana (n. s.), 28(3): 497-506.

RESUMEN. Se informan los resultados de las capturas de tábanos con trampas Malaise en la selva mediana del sur de Yucatán durante 2007. Se registraron ocho especies de la familia Tabanidae (Chrysops variegatus, Diachlorus ferrugatus, Leucotabanus canithorax, L. itzarum, Tabanus colombensis, $T$. commixtus, T. haemagogusy T. oculus) que representan $36 \%$ de las especies conocidas para este estado. Tabaninae fue la subfamilia mejor representada (tres géneros y siete especies). Las especies más abundantes fueron Tabanus commixtus y Leucotabanus itzarum.

Palabras clave: Diptera, Tabanidae, riqueza de especies, Yucatán.

Manrique-Saide, P. C., Briceño-Uc, A. R., Ibáñez-Bernal, S. \& Sandoval-Ruiz, C. A. 2012. Deer and horse flies (Diptera: Tabanidae) from the medium rainforest of southern Yucatan, Mexico. Acta Zoológica Mexicana (n. s.), 28(3): 497-506.

ABSTRACT. We report horse-flies collections with Malaise traps from the medium rainforest of Yucatan during 2007. We recorded eight species of Tabanidae (Chrysops variegatus, Diachlorus ferrugatus, Leucotabanus canithorax, L. itzarum, Tabanus colombensis, T. commixtus, T. haemagogus and T. oculus) which represented $36 \%$ of known species for this state. Tabaninae was the subfamily best represented (seven species and three genera). The most abundant species were Tabanus commixtus and Leucotabanus itzarum.

Key words: Diptera, Tabanidae, species richness, Yucatan.

\section{INTRODUCCIÓN}

La familia Tabanidae incluye 4290 especies conocidas en el mundo, de las cuales cerca de un tercio están presentes en el Neotrópico. Para México se conocen 207 especies (Fairchild \& Burger 1994) de las cuales 22 especies han sido informadas para 
el estado de Yucatán (Manrique-Saide et al. 2001). Esta familia es uno de los grupos de dípteros más estudiados en Yucatán (Ibáñez-Bernal, 1999; Manrique-Saide et al. 2001; Manrique-Saide et al. 2005); sin embargo, gran parte de los registros corresponden a áreas de la zona norte y centro del estado, quedando extensas áreas donde no existen colectas.

La zona sur del estado de Yucatán tiene gran valor biológico ya que cuenta con remanentes únicos de selva mediana en buen estado de conservación (Chablé-Santos et al. 2006). A pesar de esto, son muy pocos los estudios faunísticos realizados en esta zona y ninguno referente al orden Diptera. El objetivo del siguiente trabajo fue ampliar el conocimiento de la fauna de Tabanidae en Yucatán, mediante el inventario y análisis de la riqueza de especies de los tábanos colectados en la selva mediana de la zona sur.

\section{MATERIAL Y MÉTODOS}

Área de estudio. El estudio se llevó a cabo dentro del área natural protegida Balam

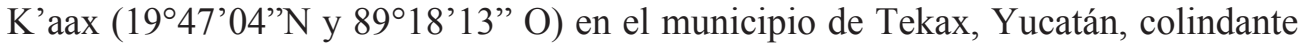
a la región PUT (Punto de UniónTerritorial) y con los estados de Campeche y Quintana Roo (figura 1). La altitud de la zona fluctúa entre los $50 \mathrm{msnm}$ en las partes más bajas y los 160 msnm en las crestas más altas (Duch 1988) y presenta un clima cálido subhúmedo con lluvias en verano o Aw“o(x’) (i)g de acuerdo con la clasificación de Köppen modificada por García et al. (1989). La temperatura media anual oscila entre los $25.1^{\circ} \mathrm{C}$ y $27{ }^{\circ} \mathrm{C}$, con un promedio anual de precipitación pluvial entre $1138.1 \mathrm{y}$ $1440 \mathrm{~mm}$ (Flores \& Espejel 1994).

El tipo de vegetación predominante en la región es selva mediana subperennifolia y se considera una zona de transición entre la selva baja caducifolia del noroeste de Yucatán y las selvas perennifolias del sur de la Península de Yucatán. La vegetación se presenta como una comunidad densa y cerrada con árboles de 15 a $40 \mathrm{~m}$ de alto con dosel y diámetros entre 20 y $80 \mathrm{~cm}$, respectivamente. Algunas de las especies dominantes representativas de este tipo de selva son: ch'i may (Acacia pennatula), kitim ché (Caesalpinia gaumeri), pixoy (Guazuma ulmofolia), zapote (Manilkara zapota) y ramón (Brosimum alicastrum) (Flores \& Espejel 1994).

Recolecta de especímenes. Se realizó mediante trampas Malaise durante los meses de enero y diciembre de 2007. Se establecieron cuatro transectos de $100 \mathrm{~m}$, formando parejas separadas por $200 \mathrm{~m}$ entre sí; cada transecto constó de tres trampas, una por cada $50 \mathrm{~m}$ (12 trampas por área de estudio, ocupando un área rectangular de $20,000 \mathrm{~m}^{2}$ ). Los organismos fueron recolectados en períodos de tres días por mes. La recolecta de cada trampa se realizó de manera individual y los especímenes se preservaron con etanol al 70\%.

Determinación taxonómica. Las muestras fueron procesadas en el Laboratorio de Entomología del Departamento de Zoología del Campus de Ciencias Biológicas 


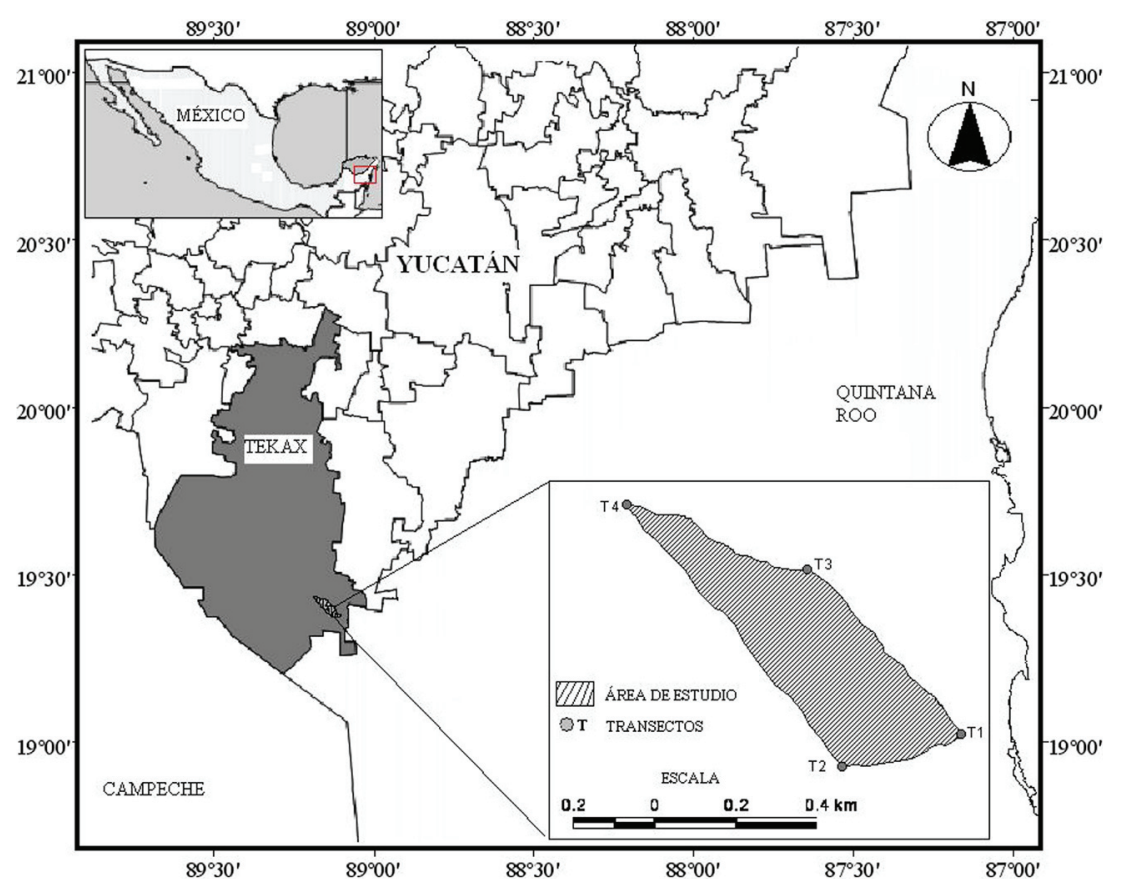

Figura 1. Ubicación geográfica del área de estudio. El recueadro en la esquina superior izquierda señala la totalidad del país (México) y en el cuadro central se representa el estado de Yucatán con los límites municipales, siendo Tekax el que está sobreado en gris. El recuadro en la parte inferior derecha señala el área de muestreo y el esquema espacial donde se establecieron los cuatro transectos.

y Agropecuarias de la Universidad Autónoma de Yucatán. Para la determinación de género y especie se utilizaron las claves de Ibáñez-Bernal (1992), y se cotejaron con los ejemplares de Tabanidae depositados en la Colección Entomológica Regional (CER) de la Universidad Autónoma de Yucatán (UADY). Una serie representativa de los ejemplares colectados fue revisada en el Instituto de Ecología A. C. Xalapa, Veracruz (INECOL), corroborando la identificación taxonómica.

Riqueza de especies. Para registrar la riqueza de especies de tábanos de la zona se consideró la sumatoria de las especies capturadas en cada una de las trampas de los cuatro sitios (Moreno 2001). Se construyó una curva de acumulación de especies para analizar la riqueza específica y para evaluar la calidad del inventario (Moreno 2001, Jiménez-Valverde y Hortal 2003). Los datos se aleatorizaron 100 veces con el programa EstimateS 8 (Colwell, 2005). Se utilizó la función de Clench: S (t) = (at) / $(1+b * t)$ y los parámetros a y $b$, posteriormente se aplicó el procedimiento de regresión no lineal con la opción de ajuste Simplex y Quasi-Newton (Jiménez-Valverde \& Hortal 2003) en el programa STATISTICA (StatSoft Inc. 2003). 


\section{RESULTADOS}

Se recolectaron 759 individuos de la familia Tabanidae pertenecientes a dos subfamilias, cuatro géneros y ocho especies. La subfamilia Tabaninae fue la mejor representada, tanto en número de especies como en ejemplares recolectados (7 especies y 758 individuos). Solamente se encontró un ejemplar perteneciente a la subfamilia Chrysopsinae (Chrysops variegatus) (Cuadro 1). Los géneros mejor representados fueron Tabanus (cuatro especies y 701 individuos) y Leucotabanus (dos especies y 51 individuos).

En la Figura 2 se observa el incremento del número de especies con relación al número de muestras. El análisis estimó un máximo de nueve especies por medio de la función de Clench. Se observa un aumento sostenido, que se estabiliza después de las 40 muestras, indicando una baja probabilidad de encontrar más especies con un mayor número de colectas. La riqueza observada representó el $89 \%$ de las especies estimadas mediante este método. Los valores obtenidos indicaron un buen ajuste al modelo $\left(\mathrm{R}^{2}=0.99778, \mathrm{a}=0.628252, \mathrm{~b}=0.069738\right.$, pendiente $\left.=0.0072\right)$.

Cuadro 1. Especies de tábanos (Tabanidae) colectados en la selva mediana del sur de Yucatán durante los meses de enero a diciembre de 2007.

\begin{tabular}{lc}
\hline \multicolumn{2}{c}{ Subfamilia } \\
\hline \multicolumn{1}{c}{ Tribu } \\
\hline \multicolumn{1}{c}{ Especie y Autor } & Número de individuos \\
\hline CHRYSOPSINAE & \\
Chrysopsini & 1 \\
Chrysops variegatus (DeGeer) & \\
TABANIDAE & 6 \\
Diachlorini & 10 \\
Diachlorus ferrugatus (Fabricius) & 41 \\
Leucotabanus canithorax Fairchild & \\
Leucotabanis itzarum (Bequaert) & 4 \\
Tabanini & 679 \\
Tabanus colombensis Macquart & 15 \\
Tabanus commixtus Walker & 3 \\
Tabanus haemagogus Williston & $\mathbf{7 5 9}$ \\
Tabanus oculus Walker & \\
Total de individuos &
\end{tabular}




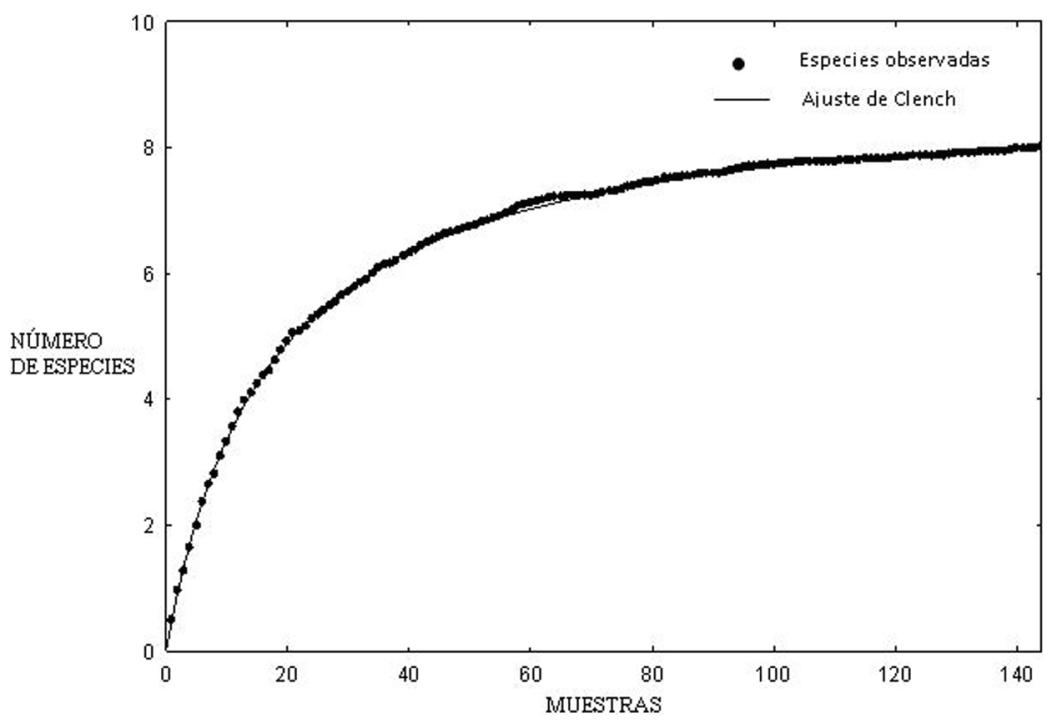

Figura 2. Curva de acumulación de especies de tábanos colectados en la selva mediana del sur de Yucatán; las muestras (puntos negros) representan cada una de las trampas Malaise durante 12 meses de muestreo. Se utilizó el modelo de Clench para el ajuste de la curva (línea sólida).

Lista anotada de las especies encontradas

\section{Chrysops variegatus (DeGeer, 1776)}

Material examinado. 1 q. México, Yucatán, PUT (N 1943'25.13”, O 89¹0'16.90”), 25-III-2007, Reyes-Cauich, T. Malaise, CER-UADY.

Comentarios. De acuerdo con Fairchild (1942, 1961, 1971 y 1986) esta especie se distribuye en toda la región Neotropical desde Chile y norte de Argentina hasta el sur de México, incluyendo las Antillas. Bequaert (1931) la refirió para Chichén Itzá, Yucatán, Ibáñez-Bernal (1992) en varias localidades de Quintana Roo y ManriqueSaide et al. (2001) para la reserva de Calakmul, Campeche y las Reservas de Ría Lagartos y Dzilam, Yucatán. También ha sido registrada en los estados de Colima, Tabasco y Veracruz (Bequaert 1931).

\section{Diachlorus ferrugatus (Fabricius, 1805)}

Material examinado. 6 q $q$. México, Yucatán, PUT (N 1943'25.13”, O 89¹0'16.90”), 25-III-2007, 27-VI-2007, Reyes-Cauich, T. Malaise, CER-UADY.

Comentarios. De acuerdo con Fairchild (1971) esta especie de distribuye desde el sur de Estados Unidos de América, Bahamas, México y América Central hasta el sur de Costa Rica. En México; Bequaert (1931) y Pearse (1945) encontraron esta especie 
en Tabasco, en Campeche y el puerto de Progreso, Yucatán. Ibáñez-Bernal (1992) la registró en las localidades de Sian Ka'an y Cancún, Quintana Roo. Manrique-Saide et al. (2001) la reportaron en diversas localidades en los tres estados de la Península de Yucatán.

\section{Leucotabanus canithorax Fairchild, 1941}

Material examinado. 10 우. México. Yucatán, PUT (N 1943’25.13”, O 89¹0'16.90”), 27-III-2007, 27-V-2007, 27-VI-2007, 18-VIII-2007, Reyes-Cauich, T. Malaise, CER-UADY.

Comentarios. Esta especie ha sido registrada en Colombia, Trinidad, Guyana, Panamá, Belice y México (Fairchild 1985; Fairchild \& Burger 1994). En la Península de Yucatán ha sido reportada en Sian Ka'an Quintana Roo (Ibáñez-Bernal 1992; Fairchild \& Burger 1994) y Calakmul Campeche (Manrique-Saide et al. 2001).

\section{Leucotabanus itzarum (Bequaert 1931)}

Material examinado. 41 우. México. Yucatán, PUT (N 1943'25.13”, O 89¹0'16.90”), 27-V-2007, 27-VI-2007, 26-VII-2007, 18-VIII-2007, Reyes-Cauich, T. Malaise, CER-UADY.

Comentarios. Esta especie es endémica de México, específicamente en los estados que conforman la Península de Yucatán (Bequaert 1931, 1933; Pearse1945; Fairchild 1971, 1985; Ibáñez-Bernal 1992; Fairchild \& Burger 1944; Manrique-Saideet al. 2001).

\section{Tabanus colombensis Macquart, 1846}

Material examinado. 4 qq. México, Yucatán, PUT (N 1943'25.13”, O 89¹0'16.90"), 21-IV-2007, 27-V-2007, 27-VI-2007, Reyes-Cauich, T. Malaise, CER-UADY.

Comentarios. Se distribuye desde Texas, Estados Unidos, hasta Brasil y Trinidad (Fairchild \& Burger 1994). En la Península de Yucatán ha sido registrada para Sian Ka'an Quintana Roo (Ibáñez-Bernal 1992), Campeche y Yucatán (Manrique-Saide et al. 2001).

\section{Tabanus commixtus Walker, 1860}

Material examinado. 679 우. México, Yucatán, PUT (N 1943’25.13”, O 89¹0’16.90”), 25-II-2007, 27-III-2007, 21-IV-2007, 27-V-2007, 19-XII-2007, Reyes-Cauich, T. Malaise, CER-UADY.

Comentarios. Se distribuye desde México hasta Venezuela, Trinidad y Martinica (Fairchild 1983; Fairchild \& Burger 1994). En la Península de Yucatán, T. commixtus fue informada en Chichen Itzá, Yucatán bajo el nombre de T. maya por Bequaert 
(1931), pero posteriormente fue registrada como T. commixtus en Sian ka'an, Quintana Roo (Ibáñez-Bernal 1992) y en varias localidades de Campeche y Yucatán (Manrique-Saide et al. 2001).

Tabanus haemagogus Williston, 1901

Material examinado. 15 우. México, Yucatán, PUT (N 1943'25.13”, O 89¹0'16.90"), 26-VII-2007, 18-VIII-2007, 17-IX-2007, Reyes-Cauich, T. Malaise, CER-UADY.

Comentarios. La distribución conocida de T. haemagogus es desde Tabasco, México, hasta Guatemala (Fairchild 1971; Fairchild \& Burger 1994). Dentro de la Península de Yucatán ha sido encontrada en Yucatán y Quintana Roo (Williston 1901; Bequaert 1931, 1933; Pearse 1938, 1945; Manrique-Saide et al. 2001).

\section{Tabanus oculus Walker, 1848}

Material examinado. 3 9. México, Yucatán, PUT (N 1943’25.13”, O 89¹0'16.90”), 27-III-2007, 27-V-2007, Reyes-Cauich, T. Malaise, CER-UADY.

Comentarios. Se distribuye en Centroamérica, desde Panamá hasta Tampico, Tamaulipas, México (Fairchild 1971; Fairchild \& Burger 1994). En la Península de Yucatán, se ha colectado en Yucatán (Bequaert 1931, 1933; Pearse 1945; ManriqueSaide et al. 2001), Quintana Roo (Bequaert 1931; Pearse 1945; Ibáñez-Bernal 1992) y Campeche (Manrique-Saide 2001).

\section{DISCUSIÓN}

Las especies recolectadas en esta zona representaron 36\% de las especies registradas en el estado de Yucatán, y $28 \%$ de las reportadas para toda la Península. Si bien las ocho especies de tábanos aquí informadas constituyen nuevos registros para la selva mediana del sur de Yucatán, no representan nuevos registros para el Estado. El grado de conservación del sur de Yucatán, así como su conexión con las selvas de Quintana Roo y Campeche sugeriría la existencia de otras especies no reportadas en estudios previos para el estado pero su presencia no pudo confirmarse en este trabajo. Hasta el momento la zona norte de Yucatán sigue siendo la de mayor riqueza de especies de Tabanidae del estado, a pesar de que estos lugares presentan un alto grado de perturbación (Ibáñez-Bernal 1999, Manrique-Saide et al. 2001, Martin-Park 2009). Sin embargo, también presenta mayor heterogeneidad en cuanto a clima, fisiografía, tipos de vegetación y uso del suelo, v.gr., manglares, selvas baja espinosa, baja caducifolia, mediana subcaducifolia y subperennifolia y vegetación acuática, con zonas inundables generalmente utilizados por los tábanos para el desarrollo de estadios tempranos) y un alta riqueza de otros grupos de animales y plantas (utilizados como fuentes de alimentación) presentes en la zona norte, en comparación con otras regiones del estado. 
Con relación a los pares de transectos sur ( 1 y 2 ) y norte ( 3 y 4 ), no se consideran variaciones importantes en el número de especies o el número de individuos, y de manera general se observaron los mismos patrones en ambas áreas. En cuanto a la representatividad de la muestra, el esfuerzo de muestreo con las trampas Malaise sugiere que se obtuvo $89 \%$ de las especies. Los estudios con trampas Malaise por lo general reportan valores de representatividad elevados; por ejemplo, Martin-Park (2009) reportó valores de representatividad comparables (83\%) utilizando este método de captura en varias áreas naturales protegidas de Yucatán. Sin embargo, tampoco reportó la presencia de especies raras a pesar de una alta representatividad de su estudio. Esto sugiere la necesidad de complementar las capturas de trampas Malaise con colectas manuales con redes y/o trampas de luz, así como atrayentes químicos $\left(\mathrm{CO}^{2}\right.$, amoniaco y ácido láctico) en estudios posteriores en esta región.

Tabanus commixtus constituyó la especie más abundante en cada uno de los transectos estudiados ( $>80 \%$ del total de individuos colectados). Esta especie se ha encontrado reiteradamente como la más abundante y con más amplia distribución en Yucatán (Manrique-Saide et al. 2001 y Martin-Park, 2009), debido probablemente a su adaptación a suelo húmedo como sitio de cría (Fairchild 1986) y sus hábitos alimentarios generalistas, que incluyen tanto animales silvestres como ganado (Fairchild 1986).

De manera general, se observa que la abundancia de tábanos aumenta significativamente al inicio del periodo de lluvias (mayo) y decrece durante los meses del periodo de "nortes" (diciembre-enero). Mucho de este patrón se explica por T. commixtus ( $>60 \%$ de las colectas durante abril y mayo) y que se ha reportado como un patrón fenológico de las poblaciones de tábanos (Fairchild 1942; Barros \& Foil 1999; Barros 2001).

En el presente estudio solo se capturaron hembras, lo cual está relacionado con las preferencias alimentarias hematófagas. Los miembros de esta familia, sean machos o hembras, se alimentan de néctar y polen, pero las hembras adicionalmente requieren consumir sangre para la producción de huevos, por lo que están en búsqueda constante de hospederos y son estimuladas por sombras de cuerpos robustos o por olores (Fairchild 1986), lo que las hace mucho más susceptibles de ser capturadas en los muestreos convencionales, como es el caso de las trampas de intercepción diseñadas por Malaise.

Si bien el inventario de la riqueza de los tábanos en Yucatán ha progresado en las últimas dos décadas, aún hay incertidumbre en lo que respecta a la presencia de especies raras que puedan estar asociadas a regiones que carecen de estudios previos, como aquellas con hábitat de agua dulce y zonas con selvas que aún permanecen conservadas (Manrique-Saide et al. 2001). Para estudios futuros se recomienda la aplicación de métodos complementarios a la trampa Malaise, con el fin de optimizar el conocimiento de fauna de tábanos en el estado. 
Agradecimientos. Este estudio fue financiado por el proyecto "Evaluación de la biodiversidad de las áreas naturales protegidas del estado de Yucatán usando grupos indicadores, propuesta de nuevas áreas y estrategias de manejo y conservación” y "Comunidades de Díptera en áreas naturales protegidas del Estado de Yucatán” del Cuerpo Académico Bio-Ecología Animal del Campus de Ciencias Biológicas y Agropecuarias, Universidad Autónoma de Yucatán.

\section{LITERATURA CITADA}

Barros, T. 2001. Seasonality and relative abundance of Tabanidae (Diptera) captured on horses in the Pantanal, Brasil. Memorias do InstitutoOswaldo Cruz, 96: 917-923.

Barros, T. \& L. Foil. 1999. Seasonal occurrence and relative abundance of Tabanidae (Diptera) from the Pantanal Region, Brasil. Memoirs on Entomology, International, 14:387-396.

Bequaert, J. 1931. Tabanidae of the Peninsula of Yucatan, Mexico, with descriptions of new species. Journal of the New York Entomological Society, 39: 533-553.

Bequaert, J. 1933. Contribution to the entomology of Yucatan. Carnegie Institute, Washington Publication, 431: 547-574.

Chablé-Santos, J., E., Gómez-Uc \& R. Pasos-Enríquez. 2006. Aves comunes del sur de Yucatán. Ediciones de la Universidad Autónoma de Yucatán. México, 137 pp.

Colwell, R. K. 2005. Estimates (Statistical stimation of species richness and shared species for samples), versión 8.0. (en línea:http://viceroy.eeb.uconn.edu/estimates)

Duch, J. 1988. La conformación territorial del Estado de Yucatán. Los componentes del medio físico. Editorial Universidad Autónoma de Chapingo. México, 427 pp.

Fairchild, G. B. 1942. The seasonal distribution of some Tabanidae (Diptera) in Panama. Annals of the Entomological Society of America, 35: 85-91.

Fairchild, G. B. 1961. Insecta Amapaensia-Diptera: Tabanidae. Studia Entomologica, 4: 433-448.

Fairchild, G. B. 1971. Family Tabanidae. Fascículo 28, pp. 1-163. In: N. Papavero (Ed.). A catalogue of the Diptera of the Americas South of the United States. Museo de Zoologia, São Paulo.

Fairchild, G. B. 1983. Notes on Neotropical Tabanidae (Diptera).XIX. The Tabanus lineola complex. Miscellaneous Publications of the Entomological Society of America, 57: 1-51.

Fairchild, G. B. 1985. Notes on Neotropical Tabanidae (Diptera) XVIII. The genus Leucotabanus Lutz. Myia, 3: 299-331.

Fairchild, G. B. 1986. The Tabanidae of Panama.Contributions of the American Entomological Institute, 22: 1-139.

Fairchild, G. B. \& J. F. Burger. 1994. A catalog of the Tabanidae (Diptera) of the America South of the United States. American Entomologist, 55: 1-249.

Flores, J. \& I. Espejel. 1994. Tipos de vegetación de la Península de Yucatán. Etnoflora Yucatanense: 3. Universidad Autónoma de Yucatán. México, 135p.

García, E., R. Vidal \& M. E. Hernández. 1989. Las regiones climáticas de México. En García de Fuentes, A. (ed.). Atlas Nacional de México, Instituto de Geografía, UNAM, vol. 2, cap. IV, núm. 10, Mapa escala 1:12 000000 .

Ibáñez-Bernal, S. 1992. Tabanidae (Diptera) de Quintana Roo, México, pp. 241-285. In: D. Navarro \& J. Robinson (Eds.). Diversidad biológica en la Reserva de la Biosfera de Sian Ka'an, Q. Roo, México. Vol. 2, México.

Ibáñez-Bernal, S. 1999. Los Díptera hematófagos y taxa relacionados de dos Áreas Protegidas del Estado de Yucatán, México (Insecta). Secretaría de Salud, Instituto Nacional de Diagnóstico y Referencia Epidemiológicos, Laboratorio de Entomología e Insectario. Informe final SNIB-CONABIO proyecto No. G011. México. (En línea, http://www.conabio.gob.mx/institucion/proyectos/resultados/InfG011.pdf). 
Jiménez-Valverde, A. \& J. Hortal. 2003. Las curvas de acumulación de especies y la necesidad de evaluar la calidad de los inventarios biológicos. Revista Ibérica de Aracnología, 8: 151-161.

Manrique-Saide, P. C., G. H. Delfín \& S. Ibáñez-Bernal. 2001. Horseflies (Diptera: Tabanidae) from protected areas of peninsula of Yucatan, Mexico. The Florida Entomologist, 84: 352-362.

Manrique-Saide, P. C., S. Ibáñez-Bernal \& I. Rodríguez-Vivas. 2005. Biología y control de moscas hematófagas de los animales domésticos, pp. 593-661 In: R. I. Rodríguez-Vivas (Ed.). Enfermedades de Importancia Económica en los Animales Domésticos. Bases epidemiológicas para su prevención, control y/o erradicación. UADY-McGraw-Hill, México.

Martin-Park, A. 2009. Diversidad de Asilidae, Syrphidae y Tabanidae (Diptera) en seis Áreas Naturales Protegidas del Estado de Yucatán, México. Tesis de Maestría. Universidad Autónoma de Yucatán. Mérida, Yucatán, México, 63 pp.

Moreno, C. E. 2001. Métodos para medir la biodiversidad. Boletín SEA, Serie Manuales y Tesis, Vol.1. Zaragoza, España, 83 pp.

Pearse, A. S. 1938. Insects from Yucatan caves. Carnegie Institute, Washington Publication, 491: 237249.

Pearse, A.S. 1945. La fauna, pp. 109-271. In: Gobierno del Estado de Yucatán (ed). Enciclopedia Yucatanense. México. Tomo I.

StatSoft, Inc. 2003. STATISTICA (data analysis software system), version 6.0 (en línea, http://www. statsoft.com).

Williston, S. W. 1901. Supplement (part), pp. 249-264. In: F. D. Godman y O. Salvin (Eds.). Biologia Centrali-Americana, United Kingdom. 